\title{
Steady uniform shear flow in a low density granular gas
}

\author{
J. J. Brey, M. J. Ruiz-Montero, and F. Moreno \\ Física Teórica, Facultad de Física, Universidad de Sevilla, Apartado de Correos 1065, E-41080 Sevilla, Spain
}

(Received 8 October 1996)

\begin{abstract}
The steady uniform shear flow of a low density granular sytem is studied by means of a kinetic model equation and also by direct Monte Carlo simulation of the Boltzmann equation. The former can be exactly solved for arbitrary shear rate and dissipation. Explicit expressions for the one-particle distribution function and the pressure tensor are obtained. Comparison of the results with those of previous theories is presented in the appropriate limits. The simulation shows that the model reproduces fairly well the values of the stresses and, in particular, the phenomenon of normal stress differences. The agreement is also very good for the velocity distribution function in the thermal velocity region, although significant discrepancies appear for large velocities. [S1063-651X(97)12803-3]
\end{abstract}

PACS number(s): 05.20.Dd, 47.50.+d, 47.20.-k

\section{INTRODUCTION}

The development of kinetic model equations describing the dynamics of particles, which collide inelastically, seems to be an important step towards the understanding of the complex phenomena taking place in rapid granular flows [1-3]. Nevertheless, the nonconservation of energy in collisions introduces quite drastic changes in the physics of the problem, and the generalization of the existing theories for ordinary fluids is far from being an easy task. This refers to both the own derivation or proposal of a kinetic equation for the distribution function of the system and also to solving the equation to obtain consistent hydrodynamic-like equations describing the macroscopic evolution. Although a hydrodynamic description, in terms of the density, the macroscopic flow velocity, and the granular temperature is suggested by analogy with normal fluids and also by experiments and computer simulations, a derivation from a more fundamental description of the system is needed.

In the last years, several kinetic equations for the oneparticle distribution function of rapid granular flows have been proposed, extending the heuristic arguments leading to the Boltzmann and Enskog equations for ordinary gases [4-7]. In addition, a more basic approach to the problem, starting from the Liouville equation, has been presented [8]. Nevertheless, even if it is assumed that these equations are relevant to the description of rapid granular flows, a fundamental difficulty arises. The standard procedure of obtaining solutions to kinetic equations is by means of the ChapmanEnskog method [9], in which an expansion in the gradients of the hydrodynamic fields is carried out. For molecular fluids, the reference state about which gradients are considered is the Maxwellian local equilibrium distribution. The corresponding reference distribution for inelastic particles is complex and has not been determined to date. As a consequence, the limit of small dissipation (i.e., the coefficient of restitution $\alpha$ close to 1) is usually considered. Nevertheless, many of the phenomena peculiar to rapid granular fluids are clearly associated to nonlinear and rheological effects and to not very small dissipation. This includes properties such as significant normal stress differences under shear $[2,10,11]$, spontaneous formation of dense clusters surrounded by re- gions of low density [12-15], and inelastic collapse [13].

The reasons mentioned above indicate that it is instructive to consider simple model kinetic equations of inelastic gases which allow controlled and detailed analysis. Kinetic models have proven to be very useful in the study of far from equilibrium states of dilute molecular gases [16]. For many physical situations, exact solutions to the models have been derived, and comparison with numerical solutions of the exact equations obtained by computer simulation shows a fairly good agreement. Very recently [8], two kinetic models for systems of inelastic hard spheres have been proposed. Both models are formulated as approximations of the revised Enskog equation, but with different quantitative accuracy. Here we will consider the limit of low densities and large length scales as compared with the diameter of the particles. In this limit, the two models lead to the same equation, and become a kinetic model of the Boltzmann equation for inelastic hard particles.

Most of the simplest far from equilibrium physical situations correspond to steady states. In this paper, we study an unbounded, steady, and uniform shear flow (USF). While in molecular fluids such a state is not possible due to viscous heating, in granular fluids this effect can be balanced by dissipation in collisions. The simplicity of the model allows us to obtain the exact solution describing the steady USF and also all the physically relevant quantities. This is done without introducing any expansion in the gradients or in the inelasticity of collisions. The results for the pressure tensor show anisotropy, i.e., normal stress differences.

It must be noted that our approach is quite different from others, in which a specific form is assumed for the oneparticle distribution function describing a given state. For instance, in the theory by Jenkins and Richman [17] a generalized Maxwellian is conjectured to model the distribution function of the steady USF. The velocity-independent coefficients are identified through the balance equation for the second order fluctuating velocity correlation tensor. This theory, which in principle also keeps all orders in the shear rate and dissipation, leads to normal stress differences which are in good agreement with the results of molecular dynamics simulations. On the other hand, the model we use has been formulated for arbitrary conditions, and no specific ap- 
proximation is introduced to apply it to the steady USF. To obtain the one-particle distribution function one has to solve the kinetic model equation under the appropriate conditions.

Another interesting approach to the same problem [18] has been carried out by expanding the Boltzmann equation to Burnett order. The perturbative expansion method is specific for the steady USF, and it is based on the observation that in this state the shear rate scales as $\sqrt{\epsilon}$, where $\epsilon=1-\alpha^{2}$. The theory results in a prediction for the normal stress differences which are very close to those of Jenkins and Richman, when the latter are expanded to the same order of approximation.

The direct Monte Carlo simulation method [19] was developped to obtain numerical solutions to the Boltzmann equation, and has been successfully applied to a great variety of situations. Since it is formulated for an arbitrary scattering law, there is no difficulty in using it to study granular flows [20]. Here we present results obtained for the steady USF, and compare them with the predictions of the model kinetic equation. For the components of the pressure tensor the agreement turns out to be very good over a wide range of values of the coefficient of restitution. Regarding the oneparticle distribution function, the agreement is only semiquantitative. This is not surprising, since the detailed Boltzmann collision operator is replaced in the model by a much simpler effective term, which is determined by the second moments of the one-particle distribution.

The structure of the paper is as follows. In Sec. II the model is briefly discussed, and the basic equations are presented. In addition, a further simplification to the formulation in Ref. [8] in introduced. It consists of an evaluation of the dissipation source term in the local equilibrium approximation. We believe this is consistent with the spirit of the model, and makes the calculations much simpler. The model kinetic equation is particularized for the USF in Sec. III, and the steady pressure tensor is computed in Sec. IV. The results are compared to the theories of Jenkins and Richman [17] and of Sela, Goldhirsch, and Noskowitz [18]. Section V deals with the one-particle distribution function, which is also compared with the above theories in the low dissipation limit. In Sec. VI we discuss the Monte Carlo simulation results and, finally, Sec. VII provides a short summary and conclusions.

\section{BASIC EQUATIONS OF THE MODEL}

We consider a gas of identical smooth disks $(d=2)$ or spheres $(d=3)$ of diameter $\sigma$ and mass $m$, whose collisions are characterized by a constant coefficient of normal restitution $\alpha$ in the interval $(0,1]$. The Boltzmann equation for the one-particle distribution function, $f(\mathbf{r}, \mathbf{v}, t)$, is $[7,8]$

$$
\left(\frac{\partial}{\partial t}+\mathbf{v}_{1} \cdot \nabla_{1}\right) f\left(\mathbf{r}_{1}, \mathbf{v}_{1}, t\right)=\mathcal{J}_{B}\left[\mathbf{r}_{1}, \mathbf{v}_{1}, t \mid f(t)\right]
$$

where $\mathcal{J}_{B}$ is the (inelastic) Boltzmann collision operator

$$
\begin{aligned}
\mathcal{J}_{B}\left[\mathbf{r}_{1}, \mathbf{v}_{1}, t \mid f(t)\right]= & \sigma^{d-1} \int d \mathbf{v}_{2} \int d \hat{\boldsymbol{\sigma}} \Theta(\mathbf{g} \cdot \hat{\boldsymbol{\sigma}})(\mathbf{g} \cdot \hat{\boldsymbol{\sigma}}) \\
& \times\left[\alpha^{-2} f\left(\mathbf{r}_{1}, \mathbf{v}_{1}^{\prime}, t\right) f\left(\mathbf{r}_{1}, \mathbf{v}_{2}^{\prime}, t\right)\right. \\
& \left.-f\left(\mathbf{r}_{1}, \mathbf{v}_{1}, t\right) f\left(\mathbf{r}_{1}, \mathbf{v}_{2}, t\right)\right] .
\end{aligned}
$$

In the above expression, $\hat{\boldsymbol{\sigma}}$ is the unit vector pointing from the center of particle 2 to the center of particle 1 at contact, $\Theta$ is the Heaviside step function, $\mathbf{g}=\mathbf{v}_{1}-\mathbf{v}_{2}$, and

$$
\begin{aligned}
& \mathbf{v}_{1}^{\prime}=\mathbf{v}_{1}-\frac{1+\alpha}{2 \alpha}(\mathbf{g} \cdot \hat{\boldsymbol{\sigma}}) \hat{\boldsymbol{\sigma}}, \\
& \mathbf{v}_{2}^{\prime}=\mathbf{v}_{2}+\frac{1+\alpha}{2 \alpha}(\mathbf{g} \cdot \hat{\boldsymbol{\sigma}}) \hat{\boldsymbol{\sigma}}
\end{aligned}
$$

are precollisional velocities leading after collision to velocities $\mathbf{v}_{1}$ and $\mathbf{v}_{2}$.

The local particle number density $n$, flow velocity $\mathbf{u}$, and temperature $T$ are defined in the usual way,

$$
\begin{gathered}
n(\mathbf{r}, t)=\int d \mathbf{v} f(\mathbf{r}, \mathbf{v}, t), \\
n(\mathbf{r}, t) \mathbf{u}(\mathbf{r}, t)=\int d \mathbf{v} \mathbf{v} f(\mathbf{r}, \mathbf{v}, t), \\
\frac{d}{2} n(\mathbf{r}, t) k_{B} T(\mathbf{r}, t)=\int d \mathbf{v} \frac{1}{2} m[\mathbf{v}-\mathbf{u}(\mathbf{r}, t)]^{2} f(\mathbf{r}, \mathbf{v}, t) .
\end{gathered}
$$

From Eq. (1) the following evolution equations for these fields are easily obtained:

$$
\begin{gathered}
\frac{\partial n}{\partial t}+\boldsymbol{\nabla} \cdot(n \mathbf{u})=0 \\
\frac{\partial \mathbf{u}}{\partial t}+\mathbf{u} \cdot \boldsymbol{\nabla} \mathbf{u}+(n m)^{-1} \boldsymbol{\nabla} \cdot \mathrm{P}=0 \\
\frac{d}{2} n k_{B} \frac{\partial T}{\partial t}+\frac{d}{2} n k_{B} \mathbf{u} \cdot \boldsymbol{\nabla} T=-(\nabla \mathbf{u}): \mathrm{P}-\boldsymbol{\nabla} \cdot \mathbf{q}-\left(1-\alpha^{2}\right) \omega
\end{gathered}
$$

where

$$
\mathrm{P}(\mathbf{r}, t)=\int d \mathbf{v} m(\mathbf{v}-\mathbf{u})(\mathbf{v}-\mathbf{u}) f(\mathbf{r}, \mathbf{v}, t)
$$

is the pressure tensor,

$$
\mathbf{q}(\mathbf{r}, t)=\int d \mathbf{v} \frac{m}{2}(\mathbf{v}-\mathbf{u})^{2} f(\mathbf{r}, \mathbf{v}, t)
$$

is the heat flux, and

$$
\begin{aligned}
\omega(\mathbf{r}, t)= & \frac{m \sigma^{d-1}}{8} \int d \mathbf{v}_{1} \int d \mathbf{v}_{2} \int d \hat{\boldsymbol{\sigma}} \Theta(\mathbf{g} \cdot \hat{\boldsymbol{\sigma}}) \\
& \times(\mathbf{g} \cdot \hat{\boldsymbol{\sigma}})^{3} f\left(\mathbf{r}, \mathbf{v}_{1}, t\right) f\left(\mathbf{r}, \mathbf{v}_{2}, t\right)
\end{aligned}
$$


is a source term describing the rate of dissipation in collisions.

Solving Eq. (1) for a given situation is a formidable task. Even in the case of ordinary gases, very little is known about solutions of the Boltzmann equation for far from equilibrium states, and the presence of dissipation complicates in a nontrivial way the structure of the Boltzmann equation. The simplest physical situation one can think of for a granular fluid is the so-called homogeneous cooling state (HCS), for which the system is uniform and the time dependence occurs entirely through the temperature. Although it has been shown $[7,21]$ that Eq. (1) admits a solution describing such state, the exact form of the distribution function is not known. For these reasons, it is worth looking for model kinetic equations similar to those which have proven to be so fruitful for ordinary fluids [16]. The basic idea is to replace the Boltzmann collision operator with a simpler form, while preserving the essential properties of the exact equation.

Very recently [8], a kinetic model along the lines sketched above has been proposed. The Boltzmann collision operator is separated into two parts: one in the velocity subspace spanned by $1, \mathbf{v}$, and $v^{2}$, and the other one in the subspace orthogonal to it. The first contribution is retained exactly, while the second one is approximated by a single relaxation time term. This guarantees that the balance equations (8)(10) are preserved by the model and that the fluxes $P$ and $\mathbf{q}$, and also the source term $w$, are the same functionals of the distribution function $f$ as in the Boltzmann equation, i.e., they are given by Eqs. (11)-(13). Since the details are described elsewhere $[8,22]$, we only give the result here. The Boltzmann collision operator is replaced by

$$
\mathcal{J}_{B} \rightarrow-\nu\left(f-f_{l}\right)-\frac{1}{n k_{B} T} f_{l} \psi(\mathbf{V})\left(1-\alpha^{2}\right) \omega
$$

where we have introduced the peculiar velocity $\mathbf{V}=\mathbf{v}-\mathbf{u}$,

$$
\psi(\mathbf{v})=\frac{m v^{2}}{d k_{B} T}-1
$$

and $f_{l}$ is the local equilibrium distribution,

$$
f_{l}(\mathbf{r}, \mathbf{v}, t)=n(\mathbf{r}, t)\left[\frac{m}{2 \pi k_{B} T(\mathbf{r}, t)}\right]^{d / 2} \exp \left[-\frac{m V^{2}(\mathbf{r}, t)}{2 k_{B} T(\mathbf{r}, t)}\right] .
$$

Also, $\nu[n(\mathbf{r}, t), T(\mathbf{r}, t)]$ is an effective local collision frequency, specified in more detail below.

The model defined by Eq. (14), although much simpler than the original Boltzmann equation, is still too complicated to allow exact calculations in most of the applications. This is due to the nonlinear functional dependence of $\omega$ on the distribution function. Then, we go a step further in the simplification, and approximate $\omega$ by its local equilibrium value, i.e.,

$$
\begin{aligned}
\omega(\mathbf{r}, t) \rightarrow & \omega_{l}(\mathbf{r}, t)=\frac{m \sigma^{d-1}}{8} \int d \mathbf{v}_{1} \int d \mathbf{v}_{2} \int d \hat{\boldsymbol{\sigma}} \Theta(\mathbf{g} \cdot \hat{\boldsymbol{\sigma}}) \\
& \times(\mathbf{g} \cdot \hat{\boldsymbol{\sigma}})^{3} f_{l}\left(\mathbf{r}, \mathbf{v}_{1}, t\right) f_{l}\left(\mathbf{r}, \mathbf{v}_{2}, t\right) \\
= & (d-1)\left(\frac{\pi}{m}\right)^{1 / 2} n^{2} \sigma^{d-1}\left(k_{B} T\right)^{3 / 2}
\end{aligned}
$$

In summary, our model kinetic equation is given by

$$
\left(\frac{\partial}{\partial t}+\mathbf{v} \cdot \boldsymbol{\nabla}\right) f=-\nu\left(f-f_{l}\right)-\frac{1}{n k_{B} T} f_{l} \psi(\mathbf{V})\left(1-\alpha^{2}\right) \omega_{l}
$$

Trivially, this equation also leads to the balance equations (8)-(10), with the only difference that in the last of them the source term $\omega$ is replaced by $\omega_{l}$. In the elastic collision limit, the kinetic model reduces to the well-known Bhatnagar-Gross-Krook (BGK) equation [23].

Application of Eq. (18) to the HCS is very simple. The solution is given by a Maxwellian with a time dependent temperature. The time evolution of this latter quantity is given by Eq. (10), which for the HCS has the solution

$$
T(t)=T(0)\left(1+\frac{t}{t_{0}}\right)^{-2}
$$

with

$$
t_{0}^{-1}=\left(1-\alpha^{2}\right) \frac{d-1}{d} \sigma^{d-1} n\left(\frac{\pi k_{B} T(0)}{m}\right)^{1 / 2} .
$$

As we have already mentioned, the exact solution of the Boltzmann equation for the HCS is not known and, in particular, it is easy to check that it is not a Maxwellian (except in the elastic limit, $\alpha=1)$. Nevertheless, both theoretical studies [7,21] and Monte Carlo simulations [20] have shown that deviations from Maxwellian are quantitatively small for thermal velocities and Eq. (19) provides a very accurate approximation for the time evolution of the temperature.

\section{UNIFORM SHEAR FLOW}

We want to study the uniform shear flow (USF), which is characterized by a constant linear velocity profile

$$
\mathbf{u}(\mathbf{r})=\mathrm{a} \cdot \mathbf{r},
$$

where $a_{i j}=a \delta_{i x} \delta_{j y}, a$ being the constant shear rate. For simplicity we consider an unbounded system. In addition, the heat flux vanishes and the density $n^{(0)}$ and temperature $T^{(0)}$ are uniform. For this flow, Eqs. (8), (9), and (10) imply that the density is constant in time, the pressure tensor is uniform, and the temperature obeys the energy balance

$$
\frac{d}{2} n^{(0)} k_{B} \frac{\partial T^{(0)}}{\partial t}=-a P_{x y}^{(0)}-\left(1-\alpha^{2}\right) \omega_{l}^{(0)} .
$$

This equation clearly shows the quite different behavior of elastic and inelastic fluids under shear. While for molecular fluids the USF is always a time-dependent state whose temperature increases monotonically in time due to viscous 
heating, in granular media the variation of the temperature arises from the balance of two opposite effects: viscosity and dissipation in collisions. As a consequence, a steady state is possible when both effects cancel each other. Our aim in the following will be to analyze such a state. It is worth mentioning that sheared granular media posses multiple steady states, depending on the initial and boundary conditions [24]. Here we restrict ourselves to the USF as described above, assuming that the system has reached such a state, and without paying any attention to its stability. A detailed analysis of these questions will be published elsewhere.

Let us introduce position and velocities coordinates with respect to a frame moving with the flow velocity $\mathbf{u}$. We define the Lagrangian coordinates by

$$
\mathbf{R}=\Lambda(t) \cdot \mathbf{r}, \quad \mathbf{V}=\mathbf{v}-\mathbf{a} \cdot \mathbf{r},
$$

where

$$
\Lambda_{i j}(t)=\delta_{i j}-a_{i j} t
$$

In terms of these, Eq. (18) becomes

$$
\begin{aligned}
& \frac{\partial f}{\partial t}+\Lambda_{i j} V_{j} \frac{\partial f}{\partial R_{i}}-a_{i j} V_{j} \frac{\partial f}{\partial V_{i}} \\
& =-\nu\left(f-f_{l}\right)-\frac{1}{n k_{B} T} f_{l} \psi\left(1-\alpha^{2}\right) \omega_{l} .
\end{aligned}
$$

Since the USF is macroscopically homogeneous in the Lagrangian frame, we expect the associated distribution function, $f^{(0)}$, to have the same property, i.e., to be independent of $\mathbf{R}$. Therefore, Eq. (25) reduces to

$$
\begin{aligned}
\frac{\partial f^{(0)}}{\partial t}-a_{i j} V_{j} \frac{\partial f^{(0)}}{\partial V_{i}}= & -\nu^{(0)}\left(f^{(0)}-f_{l}^{(0)}\right)-\frac{1}{n^{(0)} k_{B} T^{(0)}} f_{l}^{(0)} \psi^{(0)} \\
& \times\left(1-\alpha^{2}\right) \omega_{l}^{(0)}
\end{aligned}
$$

This equation implies that

$$
\frac{\partial\left\langle V_{i}\right\rangle^{(0)}}{\partial t}=-a \delta_{i x}\left\langle V_{y}\right\rangle^{(0)}
$$

where the angular brackets denote averaging with respect to $f$. Therefore, if the initial state verifies $\langle\mathbf{V}\rangle^{(0)}=0$, this property is kept in time by Eq. (26). In the following we will limit our consideration to initial conditions of this kind. This is the reason why, for velocities in the Lagrangian frame, we used the same symbol as for the peculiar velocities introduced in Sec. II. Then the local equilibrium distribution appearing in Eq. (26) has the form

$$
f_{l}^{(0)}(\mathbf{V}, t)=n^{(0)}\left(\frac{m}{2 \pi k_{B} T^{(0)}(t)}\right)^{d / 2} \exp \left(-\frac{m V^{2}}{2 k_{B} T^{(0)}(t)}\right),
$$

with all the possible time dependence occurring through the temperature.

\section{PRESSURE TENSOR}

By multiplying Eq. (26) by $V_{i} V_{j}$ and integrating over $\mathbf{V}$, it is straightforward to obtain the evolution equations for the components of the pressure tensor in the USF,

$$
\begin{aligned}
\frac{\partial}{\partial t} P_{i j}^{(0)}+a_{i k} P_{j k}^{(0)}+a_{j k} P_{i k}^{(0)}= & -\nu^{(0)}\left(P_{i j}^{(0)}-\delta_{i j} p^{(0)}\right) \\
& -\frac{2}{d} \delta_{i j}\left(1-\alpha^{2}\right) \omega_{l}^{(0)},
\end{aligned}
$$

where $p=\Sigma_{i} P_{i i} / d=n k_{B} T$ is the hydrostatic pressure. For $\alpha=1$ we recover the equations for an ordinary gas under USF in the BGK approximation, which have been extensively studied [25-27]. From Eq. (29), in particular, one obtains

$$
\begin{gathered}
\frac{\partial}{\partial t} p^{(0)}=-\frac{2}{d} a P_{x y}^{(0)}-\frac{2}{d}\left(1-\alpha^{2}\right) \omega_{l}^{(0)}, \\
\frac{\partial}{\partial t} P_{x y}^{(0)}=-a P_{y y}^{(0)}-\nu^{(0)} P_{x y}^{(0)}, \\
\frac{\partial}{\partial t} P_{y y}^{(0)}=-\nu^{(0)}\left(P_{y y}^{(0)}-p^{(0)}\right)-\frac{2}{d}\left(1-\alpha^{2}\right) \omega_{l}^{(0)} .
\end{gathered}
$$

This closed system of equations has the trivial solution $P_{x y}^{(0)}=P_{y y}^{(0)}=p^{(0)}=0$. A second steady solution, denoted by an asterisk, is given by

$$
\begin{gathered}
P_{x y}^{*}=-\frac{a}{\nu^{*}}\left[p^{*}-\frac{2}{d}\left(1-\alpha^{2}\right) \frac{\omega_{l}^{*}}{\nu^{*}}\right], \\
P_{y y}^{*}=-\frac{\nu^{*}}{a} P_{x y}^{*}, \\
\frac{a^{2}}{\nu^{*}}\left[p^{*}-\frac{2}{d}\left(1-\alpha^{2}\right) \frac{\omega_{l}^{*}}{\nu^{*}}\right]-\left(1-\alpha^{2}\right) \omega_{l}^{*}=0 .
\end{gathered}
$$

For given values of the shear rate $a$, the number density $n$, and the restitution coefficient $\alpha$, Eq. (35) determines the value of the pressure (or temperature) at which the steady state is possible. It is convenient at this point to specify the form of the effective collision frequency $\nu$. Dimensional analysis requires that

$$
\nu=\operatorname{cn} \sigma^{d-1}\left(\frac{\pi k_{B} T}{m}\right)^{1 / 2}
$$

where $c$ is a dimensionless constant. We are going to fix this by requiring that the model gives the correct (Boltzmann) value for the Navier-Stokes shear viscosity in the elastic limit $\alpha=1$. This leads to $c=16 / 5 b$, with $b \simeq 1.016$, for $d=3$ (spheres), and $c=2 / b$, with $b \simeq 1.022$, for $d=2$ (disks) [28]. When Eq. (36) is used in Eqs. (33)-(35) they reduce to

$$
\widetilde{P}_{x y}=-\left\{\frac{\left(1-\alpha^{2}\right)(d-1)}{c}\left[1-\frac{2(d-1)}{d c}\left(1-\alpha^{2}\right)\right]\right\}^{1 / 2},
$$




$$
\begin{gathered}
\widetilde{P}_{y y}=-\frac{\widetilde{P}_{x y}}{\widetilde{a}}=1-\frac{2(d-1)}{d c}\left(1-\alpha^{2}\right), \\
\widetilde{a}^{2}=\frac{d(d-1)\left(1-\alpha^{2}\right)}{d c-2(d-1)\left(1-\alpha^{2}\right)} .
\end{gathered}
$$

We have introduced the dimensionless pressure tensor $\widetilde{P}_{i j}$ and shear rate $\tilde{a}$ in the steady state as

$$
\widetilde{P}_{i j}=\frac{P_{i j}^{*}}{p^{*}}, \quad \tilde{a}=\frac{a}{\nu^{*}} .
$$

Equation (39) shows that in the steady state the ratio of the shear rate to the collision frequency depends only on the restitution coefficient. The simple dependence of the presure tensor on the shear rate found here contrasts with the complex one found in the time-dependent uniform shear flow of ordinary gases [25-27]. Let us suppose we carry out a series of experiences, all of them in the same system, i.e., with given values of $\alpha$ and $n$, but with different values of the shear rate, measuring the shear viscosity in the steady state. Taking into account that Eq. (39) implies that $T^{*} \propto a^{2}$, it follows from Eq. (37) that the generalized shear viscosity $\eta^{*}$ is linear in the shear rate,

$$
\eta^{*}(a) \equiv-\frac{P_{x y}^{*}}{a} \propto a .
$$

Of course, the proportionality constant in this expression depends on the coefficient of restitution.

The other components of the pressure tensor are easily computed by means of Eq. (29), particularized for the steady state. One obtains

$$
\widetilde{P}_{x x}=1+\frac{2(d-1)^{2}\left(1-\alpha^{2}\right)}{d c}
$$

and, for a three-dimensional system,

$$
\widetilde{P}_{z z}=\widetilde{P}_{y y}, \quad \widetilde{P}_{x z}=\widetilde{P}_{y z}=0
$$

Let us note that the reduced pressure tensor in the steady state is expressed as a function of $\alpha$ alone, being independent, in particular, of the applied shear rate. Then, for instance, for the normal stress ratios we have

$$
\begin{gathered}
\frac{P_{x x}^{*}}{P_{y y}^{*}}=\frac{d c+2(d-1)^{2}\left(1-\alpha^{2}\right)}{d c-2(d-1)\left(1-\alpha^{2}\right)}, \\
\frac{P_{y y}^{*}}{P_{z z}^{*}}=1 .
\end{gathered}
$$

Therefore, the model predicts anisotropy of the diagonal terms of the pressure tensor in the shear plane, but not in the plane perpendicular to the flow. The existence of normal stress differences in shear flows of normal fluids is very well known. It is a viscometric effect which appears beyond the Navier-Stokes approximation [29]. For a low density USF they have been calculated using the BGK model [27]. There, differences between $P_{x x}$ and $P_{y y}$ but not between $P_{y y}$ and $P_{z z}$ were found.

As we have already mentioned, an approximated theory for the steady USF of smooth inelastic disks has been formulated by Jenkins and Richman [17]. Their results for the second velocity moments in the low density limit read, in our notation,

$$
\begin{gathered}
\widetilde{a}^{(J R) 2}=\frac{(1+\alpha)\left(1-\alpha^{2}\right)(7-3 \alpha)^{2}}{c^{2}(9+7 \alpha)}, \\
\widetilde{P}_{x y}^{(J R)}=-\frac{4}{25-9 \alpha}[(9+7 \alpha)(1-\alpha)]^{1 / 2}, \\
\widetilde{P}_{y y}^{(J R)}=\frac{9+7 \alpha}{25-9 \alpha}, \\
\widetilde{P}_{x x}^{(J R)}=\frac{41-25 \alpha}{25-9 \alpha} .
\end{gathered}
$$

Although these expressions may appear rather different from the corresponding results obtained from our model kinetic equation, Figs. 1 and 2 show that the discrepancies are quite small for low dissipation, and tend to vanish when $\alpha$ approaches unity. Let us also mention that recent molecular dynamics simulations [24] have found that the values of the steady temperature for a dilute gas of inelastic disks can be closely fitted by an empirical relation, which in our units reads

$$
\tilde{a}^{-2}=\frac{16 A c^{2}}{\pi}\left(\frac{1}{1-\alpha^{2}}+B\right),
$$

with $A \simeq 0.080$ and $B \simeq-0.54$. The above expression has the same functional dependence on the restitution coefficient as Eq. (39). In addition, from this latter equation one identifies $A \simeq 0.100$ and $B \simeq-0.511$, which are surprisingly close to the simulation values, taking into account that no excluded volume effects are considered in our model, and also that the flow observed in Ref. [24] is highly nonuniform and it exhibits time-dependent microstructures.

Sela, Goldhirsch, and Noskowitz [18] carried out a study of a two-dimensional granular USF to Burnett order using the Boltzmann equation. They arrived at expressions for the steady temperature and the pressure tensor of the forms

$$
\begin{gathered}
\widetilde{a}^{2}=C\left(1-\alpha^{2}\right)+D\left(1-\alpha^{2}\right)^{3 / 2}+O\left(1-\alpha^{2}\right)^{2}, \\
\widetilde{P}_{x y}=E\left(1-\alpha^{2}\right)^{1 / 2}+O\left(1-\alpha^{2}\right)^{3 / 2}, \\
\widetilde{P}_{x x}=1+F\left(1-\alpha^{2}\right)+O\left(1-\alpha^{2}\right)^{2}, \\
\widetilde{P}_{y y}=1+G\left(1-\alpha^{2}\right)+O\left(1-\alpha^{2}\right)^{2} .
\end{gathered}
$$

Here $C, D, E, F$, and $G$ are dimensionless constants. In Table I we compare the values for these constants given in Ref. [18] with those obtained by expanding the expressions derived in this section. Also given are the results obtained from the expansion of the Jenkins and Richman theory. The values from the three theories are very close and, in particu- 


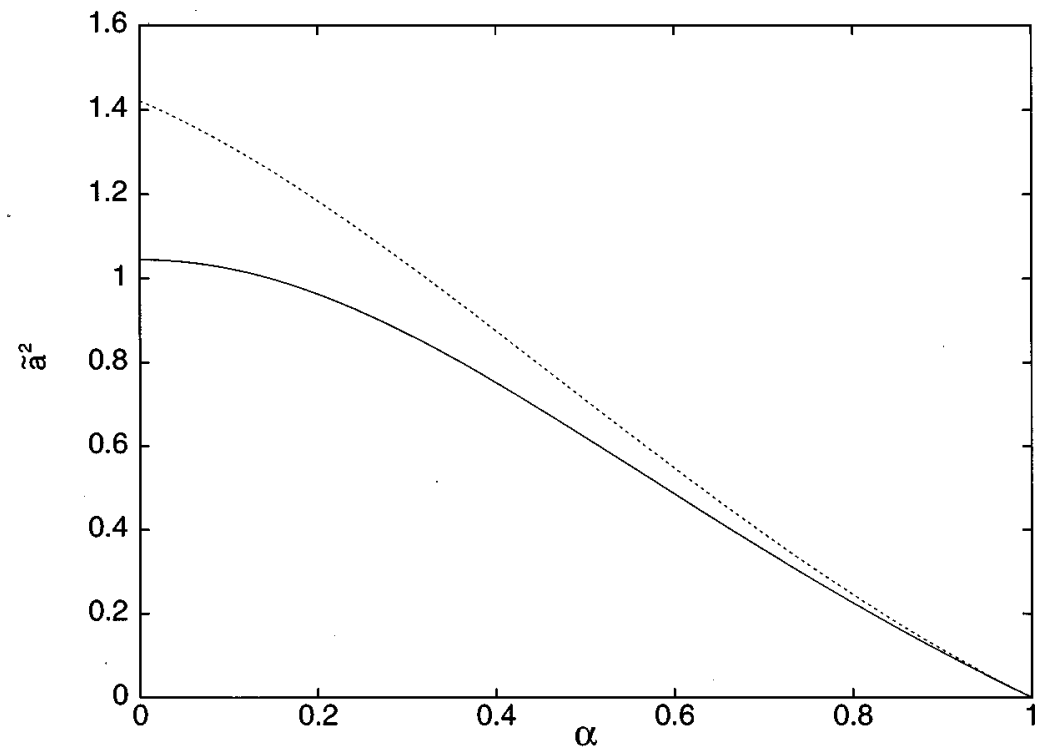

FIG. 1. Reduced shear rate $\widetilde{a}$ as a function of the coefficient of restitution $\alpha$ for a twodimensional steady USF. The solid line corresponds to the present theory, and the dashed line to that of Jenkins and Richman. lar, the agreement between our results and the Burnett expansion of the Boltzmann equation is excellent.

$$
s \equiv \int_{0}^{t} d t^{\prime} \nu^{(0)}\left(t^{\prime}\right)
$$

\section{VELOCITY DISTRIBUTION FUNCTION}

The formal solution of Eq. (26) can be written as

$$
\begin{aligned}
f^{(0)}(\mathbf{V}, t)= & e^{-s} e^{t a_{i j} V_{j} \frac{\partial}{\partial V_{i}} f^{(0)}(\mathbf{V}, 0)} \\
& +\int_{0}^{t} d t^{\prime} e^{-\left(s-s^{\prime}\right)} e^{\left(t-t^{\prime}\right) a_{i j} V_{j}\left(\partial / \partial V_{i}\right)}\left[\nu^{(0)}\left(t^{\prime}\right)\right. \\
& \left.-\frac{1}{n^{(0)} k_{B} T^{(0)}\left(t^{\prime}\right)} \psi^{(0)}\left(\mathbf{V}, t^{\prime}\right)\left(1-\alpha^{2}\right) \omega_{l}^{(0)}\left(t^{\prime}\right)\right] \\
& \times f_{l}^{(0)}\left(\mathbf{V}, t^{\prime}\right)
\end{aligned}
$$

where is a measure of the average number of collisions per particle between 0 and $t, s^{\prime}=s\left(t^{\prime}\right)$, and $\exp \left[t a_{i j} V_{j}\left(\partial / \partial V_{i}\right)\right]$ is a shift operator in velocity space,

$$
e^{t a_{i j} V_{j}\left(\partial / \partial V_{i}\right)} h(\mathbf{V})=h[\Lambda(-t) \cdot \mathbf{V}]
$$

Of course, the time-dependent temperature in Eq. (55) must be consistently calculated, i.e., it is given by the solution of Eqs. (30)-(32). Let us now consider the long time limit of $f^{(0)}(t)$. Assuming that the steady state discussed in Sec. IV is reached, we know that the temperature tends to the constant value given by Eq. (39), and $s$ diverges. The conclusion is that $f^{(0)}(\mathbf{V}, t)$ approaches the steady form

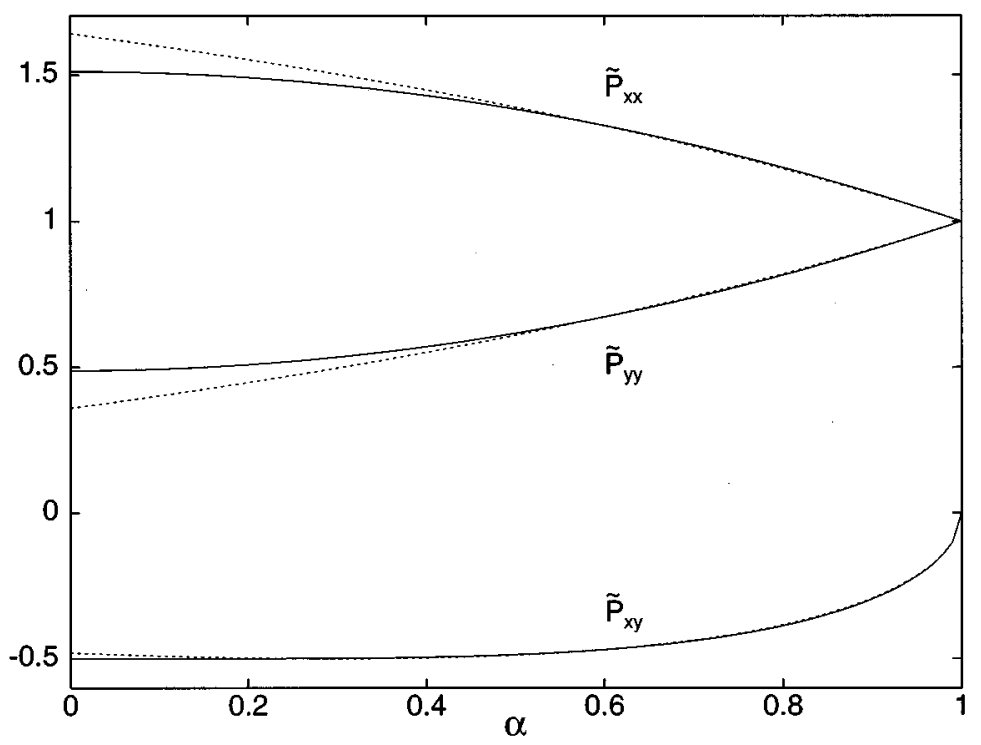

FIG. 2. The pressure tensor for a twodimensional steady USF. The solid lines correspond to the present theory, and the dashed ones to that of Jenkins and Richman. 
TABLE I. Comparison of the values of the coefficients in expansions given by Eqs. (51)-(54) obtained with different theories and in the present work.

\begin{tabular}{lccc}
\hline \hline & Model & $\begin{array}{c}\text { Sela, Goldhirsch, } \\
\text { and Noskowitz }\end{array}$ & Jenkins and Richman \\
\hline$C$ & 0.511 & 0.511 & 0.522 \\
$D$ & 0 & 0 & 0 \\
$E$ & -0.714 & -0.714 & -0.707 \\
$F$ & 0.511 & 0.522 & 0.5 \\
$G$ & 0.511 & 0.522 & 0.5 \\
\hline \hline
\end{tabular}

$$
\begin{aligned}
f^{*}(\mathbf{V})= & \int_{0}^{\infty} d t e^{-\nu^{*} t} e^{t a_{i j} V_{j}\left(\partial / \partial V_{i}\right)} \\
& \times\left[\nu^{*}-\frac{1}{n^{(0)} k_{B} T^{*}} \psi^{*}(\mathbf{V})\left(1-\alpha^{2}\right) \omega_{l}^{*}\right] f_{l}^{*}(\mathbf{V}),
\end{aligned}
$$

with

$$
\psi^{*}(\mathbf{V})=\frac{m V^{2}}{d k_{B} T^{*}}-1
$$

It can be checked that this expression reproduces the values of the steady pressure tensor found in Sec. IV, and also that it gives a vanishing heat flux. Now we introduce dimensionless quantities by

$$
\begin{gathered}
\tilde{\mathbf{V}}=\left(\frac{2 k_{B} T^{*}}{m}\right)^{-1 / 2} \mathbf{V}, \\
\widetilde{f}=\frac{1}{n^{(0)}}\left(\frac{2 k_{B} T^{*}}{m}\right)^{d / 2} f^{*} .
\end{gathered}
$$

Then Eq. (58) reduces to

$$
\begin{aligned}
\widetilde{f}(\tilde{\mathbf{V}})= & \pi^{-d / 2} \int_{0}^{\infty} d s e^{-s} e^{s \tilde{a} \tilde{V}_{y}\left(\partial / \partial \tilde{V}_{x}\right)} \\
& \times\left[1-\frac{d-1}{c}\left(\frac{2}{d} \widetilde{V}^{2}-1\right)\left(1-\alpha^{2}\right)\right] e^{-\widetilde{V}^{2}},
\end{aligned}
$$

where use has been made of Eqs. (17) and (36). In the case of a system of hard spheres, the marginal distribution for the $z$ component of the velocity is easily obtained,

$$
\begin{aligned}
\widetilde{f}_{z}\left(\widetilde{V}_{z}\right) \equiv & \int_{-\infty}^{\infty} d \widetilde{V}_{x} \int_{-\infty}^{\infty} d \widetilde{V}_{y} \widetilde{f}(\widetilde{\mathbf{V}})=\pi^{-1 / 2} \\
& \times\left[1-\frac{2\left(1-\alpha^{2}\right)}{3 c}\left(2 \widetilde{V}_{z}^{2}-1\right)\right] e^{-\widetilde{V}_{z}^{2}}
\end{aligned}
$$

This expression shows a limitation of our model kinetic equation, namely, that the distribution function takes unphysical negative values for large enough velocities. Nevertheless, the velocity values for which Eq. (63) is negative are given by

$$
V_{z}^{2}>\left[\frac{3 c}{2\left(1-\alpha^{2}\right)}+1\right] \frac{k_{B} T^{*}}{m},
$$

and, therefore, the distribution function is positive in all the range of thermal velocities, even in the limit $\alpha \rightarrow 0$. It is then possible that Eq. (62) provides an accurate approximation of the solution of the Boltzmann equation in all the relevant region of the distribution function. This point will be analyzed in detail in Sec. VI.

The marginal velocity distribution in the shear plane, also for $d=3$, is

$$
\begin{aligned}
\widetilde{f}_{x y}\left(\widetilde{V}_{x}, \widetilde{V}_{y}\right) \equiv & \int_{-\infty}^{\infty} d \widetilde{V}_{z} \widetilde{f}(\widetilde{\mathbf{V}}) \\
= & \pi^{-1} \int_{0}^{\infty} d s e^{-s} e^{-\left[\left(\widetilde{V}_{x}+s \tilde{a} \tilde{V}_{y}\right)^{2}+\widetilde{V}_{y}^{2}\right]} \\
& \times\left\{1-\frac{4\left(1-\alpha^{2}\right)}{3 c}\left[\left(\widetilde{V}_{x}+s \tilde{a} \widetilde{V}_{y}\right)^{2}+\widetilde{V}_{y}^{2}\right]\right\} .
\end{aligned}
$$

In order to compare with previous theories, we have carried out a perturbative expansion of $\widetilde{f}$ for $d=2$ in powers of $1-\alpha^{2}$. The result is

$$
\begin{aligned}
\widetilde{f}(\widetilde{\mathbf{V}})= & \pi^{-1} e^{-\widetilde{V}^{2}}\left[1-\frac{\widetilde{V}^{2} \sin 2 \theta}{\sqrt{c}}\left(1-\alpha^{2}\right)^{1 / 2}\right. \\
& +\left(1-2 \widetilde{V}^{2}+\widetilde{V}^{2} \cos 2 \theta+\frac{1}{2} \widetilde{V}^{4}-\frac{1}{2} \widetilde{V}^{4} \cos 4 \theta\right) \\
& \left.\times \frac{1-\alpha^{2}}{c}+O\left(1-\alpha^{2}\right)^{3 / 2}\right]
\end{aligned}
$$

Here, we have introduced a polar representation for the velocity, $\widetilde{\mathbf{V}}(\widetilde{V}, \theta)$, where $\theta$ is measured with respect to the streaming direction $x$.

Jenkins and Richman [17] assumed that the steady distribution function has the form of a generalized Gaussian,

$$
\widetilde{f}^{(\mathrm{JR})}=\frac{1}{\pi \sqrt{|\mathrm{K}|}} \exp \left(-\tilde{\mathbf{V}} \cdot \mathrm{K}^{-1} \cdot \tilde{\mathbf{V}}\right)
$$

where $\mathrm{K}$ is a matrix which accounts for the anisotropy existing in the USF. When the low density limit of the above distribution is considered and the result expanded to order $\left(1-\alpha^{2}\right)$ one obtains

$$
\begin{aligned}
\widetilde{f}^{(\mathrm{JR})}(\widetilde{\mathbf{V}})= & \pi^{-1} e^{-\widetilde{V}^{2}}\left[1-\frac{\widetilde{V}^{2} \sin 2 \theta}{\sqrt{2}}\left(1-\alpha^{2}\right)^{1 / 2}\right. \\
& +\left(1-2 \widetilde{V}^{2}+2 \widetilde{V}^{2} \cos 2 \theta+\frac{1}{2} \widetilde{V}^{4}-\frac{1}{2} \widetilde{V}^{4} \cos 4 \theta\right) \\
& \left.\times \frac{1-\alpha^{2}}{4}+O\left(1-\alpha^{2}\right)^{3 / 2}\right]
\end{aligned}
$$

If we neglect the difference between $c(\sim 1.96)$ and 2 , both expressions agree to order $\left(1-\alpha^{2}\right)^{1 / 2}$. The terms of order $\left(1-\alpha^{2}\right)$ have the same dependence on the velocity modulus 


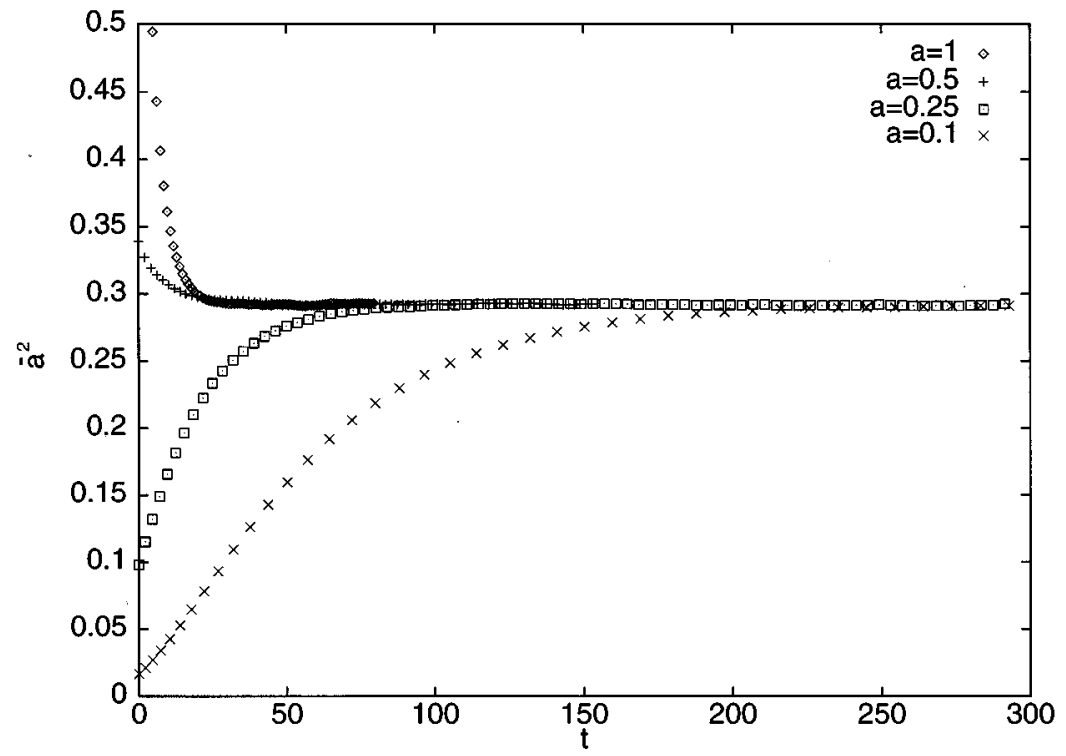

FIG. 3. Time evolution of the reduced shear rate $\bar{a}$ for $\alpha=0.8$, and different values of the shear rate $a$. In all cases, the initial distribution was homogeneous with a Maxwellian velocity distribution in the Lagrangian frame. Time is measured in units of $c(2 \sqrt{\pi} \nu(0))^{-1}$.

and also on $\theta$, but all the constant prefactors differ. In spite of this difference, let us notice that both expressions (66) and (68) make the same contribution to the diagonal part of the pressure tensor when the constant $c$ is again approximated by 2 . This can be verified by a direct calculation (see also Table I).

The distribution function for the USF of a twodimensional granular gas has also been calculated at the same order by Sela, Goldhirsch, and Noskowitz in Ref. [18]. There, a much more complicated dependence on both polar components of the velocity is found. By comparing with Jenkins and Richman results, the authors conclude that the constants appearing as prefactors for the second harmonics in $\theta$ in Eq. (68) can be considered as rough averages of the corresponding functions obtained by them. We refer the reader to their paper for details.

\section{DIRECT MONTE CARLO SIMULATION}

The direct simulation Monte Carlo method [19] has proved to be a very useful tool to obtain numerical solutions of the Boltzmann equation for molecular fluids. Very recently, it has also been applied to study the HCS of a low density granular flow [20]. Since the details of the method have been extensively discussed in Ref. [19], they will not be given here.

We saw in Sec. III that the distribution function for the USF becomes homogeneous in the Lagrangian frame. The consideration of homogeneous states allows a great simplification of the simulation. Therefore, we have carried out the simulation in the Lagrangian frame and restricted ourselves to solutions of the Boltzmann equation which stay homogeneous in that frame. In other words, we numerically solved the equation [compare with Eq. (26)]

$$
\frac{\partial f^{(0)}}{\partial t}-a_{i j} V_{j} \frac{\partial f^{(0)}}{\partial V_{i}}=\mathcal{J}_{B}\left[\mathbf{V} \mid f^{(0)}\right]
$$

Of course, this implies that the possibility of spontaneous formation of spatial inhomogeneities is eliminated in the

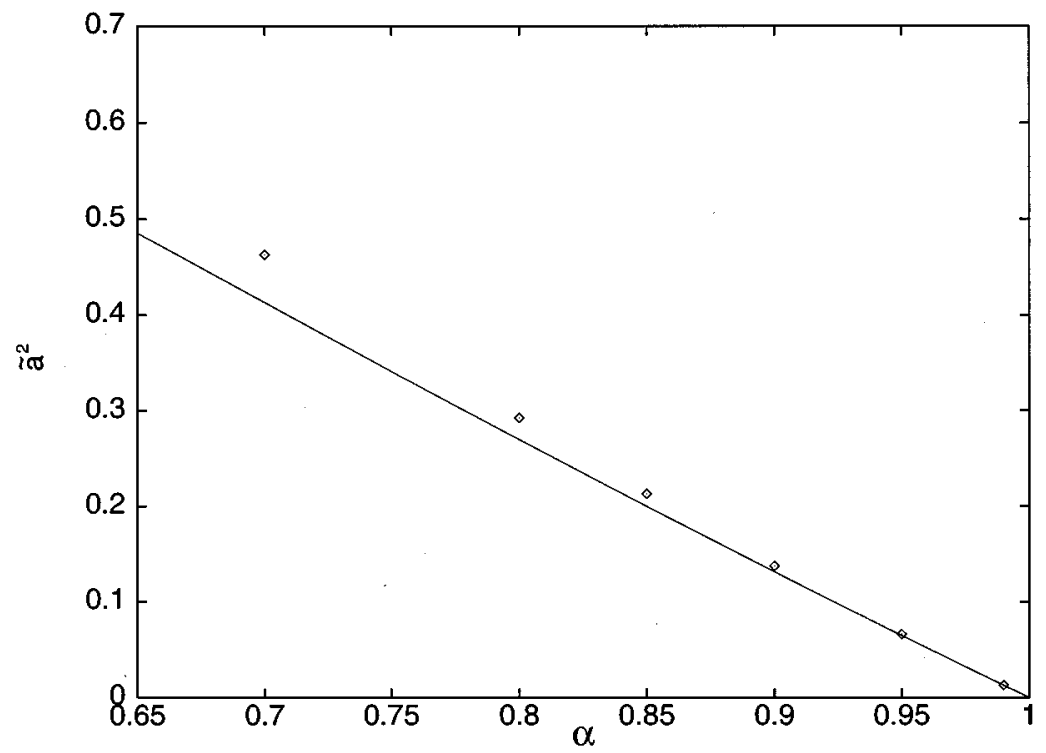

FIG. 4. Steady reduced shear rate $\tilde{a}$ as a function of the coefficient of restitution $\alpha$ for a dilute system of hard disks. The solid line corresponds to the kinetic model, and the symbols are results from the Monte Carlo simulation. 


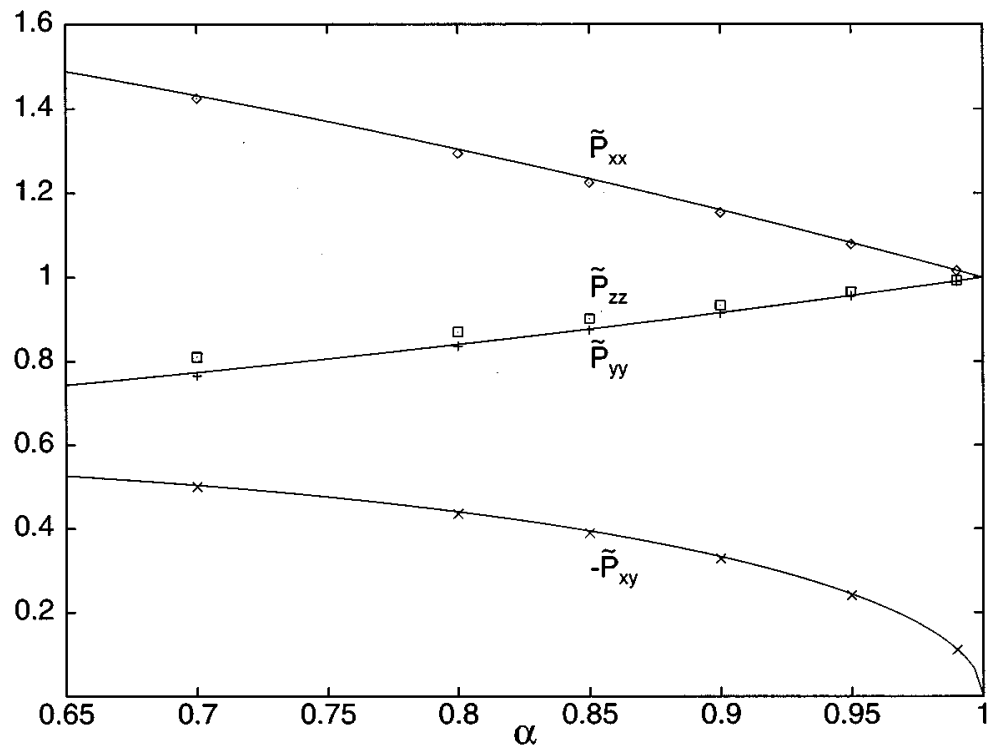

FIG. 5. The same as in Fig. 4 for the reduced pressure tensor $\widetilde{P}_{i j}$.

simulation. Our aim here is to study the properties of the homogeneous steady state. Its stability will be analyzed elsewhere.

For homogeneous systems there is no need to split the system into cells and, consequently, the spatial coordinates of the particles do not play any role in the simulation. In addition, no boundary conditions must be introduced. In our simulation we have considered a three-dimensional system. The number of particles is $N=1000$, and the results have been averaged over 500 different trajectories. The time interval $\Delta t$ over which it is assumed that free motion, including the effect of the inertial force, and collisions, are uncoupled has been taken $\delta t=0.025 \nu_{0}^{-1}$, where $\nu_{0}=(2 \sqrt{\pi} / c) \nu(t)$, with $\nu(t)$ being the instantaneous value of the collision frequency given by Eq. (36). We considered a nonconstant time step in order to guarantee that it always remains much smaller than the average time between collisions, in spite of the change of the temperature [30]. The initial velocity distribution in all cases is a Maxwellian.
In Fig. 3 we present the time evolution of $\bar{a} \equiv a / \nu(t)$ for $\alpha=0.8$ and four different values of the shear rate, namely, $a=0.1,0.25,0.5$, and 1 . Time is measured in units of $c(2 \sqrt{\pi} \nu(0))^{-1}$, where $\nu(0)$ is the initial collision frequency. After an initial transient period, all curves converge to the same steady value, as predicted by Eq. (39). The same qualitative behavior has been found for all the reduced components of the pressure tensor $P_{i j}(t) / p(t)$. Therefore, in the following we will concentrate on the dependence of the steady values of the reduced quantities on the restitution coefficient $\alpha$, once we have checked they do not depend on the shear rate.

The results obtained for the steady reduced shear rate $\tilde{a}$ for different values of $\alpha$ are shown in Fig. 4. The statistical errors are smaller than the symbols used to represent the data. Also plotted is the prediction of our model kinetic equation, i.e. Eq. (39) with $d=3$. It is seen that the agreement is remarkable at low dissipation, although the discrepancy increases as the restitution coefficient decreases. We

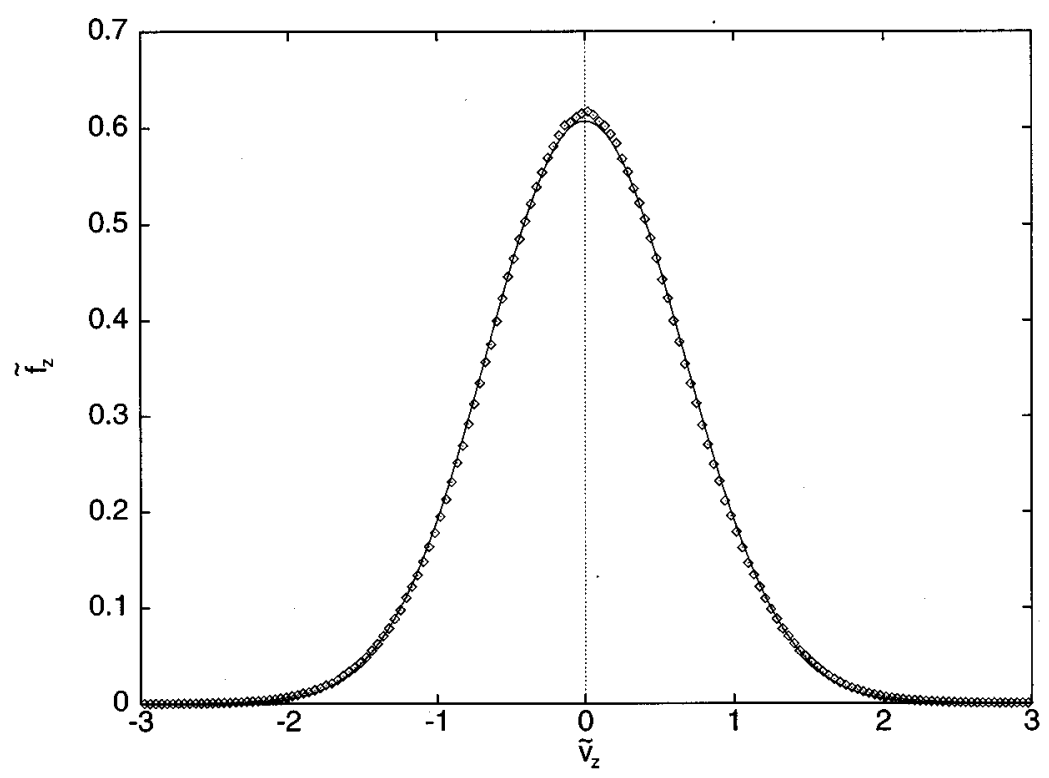

FIG. 6. Marginal velocity distribution function in the direction perpendicular to the shear plane. The symbols are simulation data, and the solid line corresponds to the model kinetic equation. The restitution coefficient is $\alpha=0.8$. 


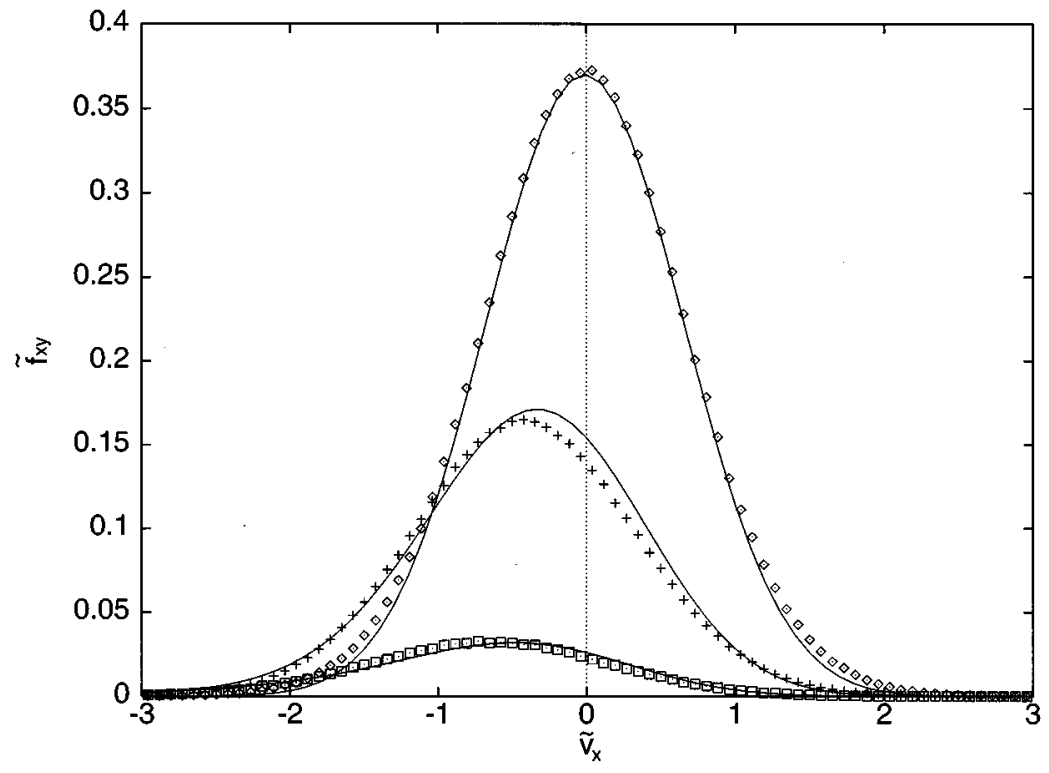

FIG. 7. Marginal velocity distribution in the shear plane. The symbols are simulation data, and the solid lines results from the kinetic model. The distributions are shown vs $\widetilde{V}_{x}$ for three different given values of $\widetilde{V}_{y}$, namely, $\widetilde{V}_{y}=0.019,0.75$, and 1.37 , from top to bottom. The coefficient of restitution is $\alpha=0.8$. have verified that all the numerical data are fitted if one takes $c \approx 2$ in Eq. (39). Therefore, it is tempting to conclude that the functional form given by the model is correct, and that the observed discrepancy follows from the value given to the constant in the collision frequency. Our choice emphasizes the role of the shear viscosity. In Fig. 5, we present the measured values for the components of the pressure tensor and compare them to the model expressions, Eqs. (37), (38), (42), and (43). The theory reproduces very well the results for the normal stress $\widetilde{P}_{x x}$ and the shear stress $\widetilde{P}_{x y}$. On the other hand, the simulation shows that there is anisotropy in the plane perpendicular to the flow velocity, being $\widetilde{P}_{z z}>\widetilde{P}_{y y}$, while the kinetic model leads to $\widetilde{P}_{y y}=\widetilde{P}_{z z}$. The same kind of anisotropy has been found in molecular dynamics simulations of shear flows at low density, although the inequality changes in sign at high densities [11,31]. It is observed that the theoretical values are in between the two numerical ones, as expected for consistency. Nevertheless, let us note that the relative normal stress differences in this plane are about $3 \%$ for $\alpha=0.7$, and that they decrease as the restitution coefficient increases. We conclude that the model kinetic equation we are considering gives a fairly good approximation of the pressure tensor for a granular dilute gas under steady USF.

Let us now check at what extension the above conclusion also holds for the velocity distribution function. We have verified that, once the steady state has been reached, the shape of the distribution is determined only by the coefficient of restitution, according to the predictions of the model. Figure 6 depicts the marginal distribution $\widetilde{f}_{z}\left(\widetilde{V}_{z}\right)$ for $\alpha=0.8$. The agreement is quite good in the velocity range shown, although a discrepancy is observed in the height of the maximum at $\widetilde{V}_{z}=0$. In addition, the agreement becomes much worse if larger velocities are considered. In fact, one cannot expect a single relaxation approximation to be able to accurately describe velocities beyond the thermal region, since

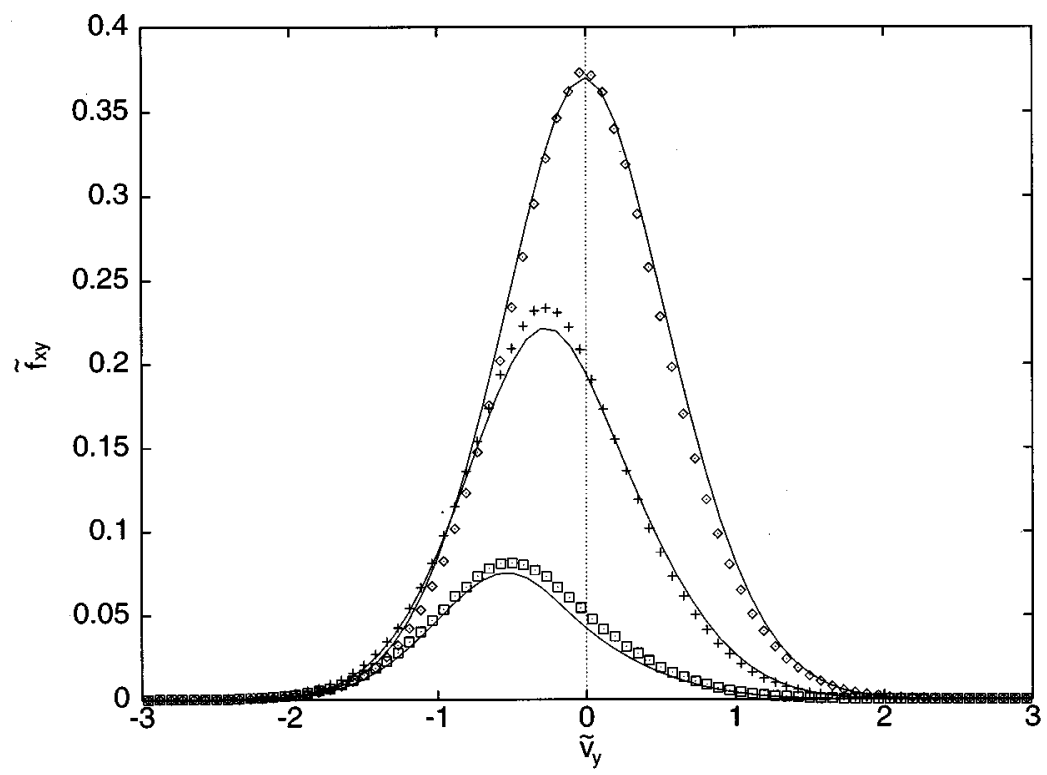

FIG. 8. Marginal velocity distribution in the shear plane. The symbols are simulation data, and the solid lines results from the kinetic model. The distributions are shown vs $\widetilde{V}_{y}$ for three different given values of $\widetilde{V}_{x}$, namely, $\widetilde{V}_{x}=0.019,0.75$, and 1.37 , from top to bottom. The coefficient of restitution is $\alpha=0.8$. 
the evolution of the distribution function is given in terms of only the first five moments. The same comment applies to the results presented in Figs. 7 and 8. There, the marginal distribution function $\widetilde{f}_{x y}\left(\widetilde{V}_{x}, \widetilde{V}_{y}\right)$ is plotted as a function of $\widetilde{V}_{y}$ for several fixed values of $\widetilde{V}_{x}$ and as a function of $\widetilde{V}_{x}$ for fixed values of $\widetilde{V}_{y}$, respectively. Again, all distributions correspond to $\alpha=0.8$. It is clearly observed that the asymmetry of the distribtion function increases as the distance from the absolute maximum increases.

\section{CONCLUSIONS}

The steady state of USF for a low density granular flow has been studied by means of a kinetic model, and also by using the direct Monte Carlo simulation technique. The simple form of the collision term in the model allows the construction of an exact explicit analytical solution, without restriction to small shear rate or low dissipation. Therefore, no other scale separation than those implicit in the own kinetic equation is assumed. To Burnett order, the results for the pressure tensor are very close to those recently derived by expanding the Boltzmann equation for a system of hard disks [18]. They also agree with the predictions of the Jenkins and Richman theory [17], which is based on the hypothesis that the distribution function can be written as a generalized Gaussian.
Comparison with the simulation data shows that the model describes fairly well the dependence of the pressure tensor, including the normal stress differences, on the coefficient of restitution. Regarding the complete velocity distribution, the model gives accurate results for small velocities, but significant discrepancies appear in the high-velocity region. This is consistent with the expectation that a BGK-like model is a good approximation of low moments of the distribution, but fails for high moments. Let us note that any perturbative approximation of the Boltzmann equation for a nonequilibrium situation is also expected to break down for large enough velocities.

Finally, let us mention that the work presented here can be extended in both aspects, modeling and simulation, to the revised Enskog equation [8], where many of the phenomena mentioned in Sec. I, and that occur at higher density, can be studied. Also, the very interesting problem of the stability of the steady USF state considered here and the possible existence of other steady states can be addressed.

\section{ACKNOWLEDGMENTS}

This research was partially supported by Grant No. PB960534 from the Dirección General de Investigación Científica y Técnica (Spain).
[1] P. K. Haff, J. Fluid Mech. 134, 401 (1983).

[2] C. S. Campbell, Ann. Rev. Fluid Mech. 22, 57 (1990).

[3] H. M. Jaeger and S. R. Nagel, Science 255, 1523 (1992); H. M. Jaeger, S. R. Nagel, and R. P. Behringer, Phys. Today 49(4), 32 (1996).

[4] J. T. Jenkins and S. B. Savage, J. Fluid Mech. 130, 187 (1983).

[5] C. Lun, S. Savage, D. Jeffrey, and N. Chepurnuy, J. Fluid Mech. 140, 223 (1984).

[6] J. T. Jenkins and M. W. Richman, Arch. Rat. Mech. 87, 355 (1985); Phys. Fluids 28, 3485 (1985).

[7] A. Goldsthein and M. Shapiro, J. Fluid Mech. 282, 75 (1995).

[8] J. J. Brey, J. W. Dufty, and A. Santos, J. Stat. Phys. (to be published); J. W. Dufty, J. J. Brey, and A. Santos, Physica (to be published).

[9] J. A. McLennan, Introduction to Nonequilibrium Statistical Mechanics (Prentice-Hall, Englewood Cliffs, NJ, 1989).

[10] O. R. Walton and R. L. Braun, Acta Mech. 63, 73 (1986).

[11] M. A. Hopkins and H. S. Shen, J. Fluid Mech. 244, 477 (1992).

[12] B. Bernu and R. Mazighi, J. Phys. A 23, 5745 (1990).

[13] S. McNamara and W. R. Young, Phys. Fluids A 4, 496 (1992); 5, 34 (1993); Phys. Rev E 53, 5089 (1996).

[14] N. Sela and I. Goldhirsch, Phys. Fluids A 7, 507 (1995).

[15] I. Goldhirsch and G. Zanetti, Phys. Rev. Lett. 70, 1619 (1993).

[16] For a recent review, see J. W. Dufty, in Lectures on Thermodynamics and Statistical Mechanics, edited by M. López de Haro and C. Varea (World Scientific, Singapore, 1990), pp. $166-181$.
[17] J. T. Jenkins and M. W. Richman, J. Fluid Mech. 192, 313 (1988).

[18] N. Sela, I. Goldhirsch, and S. N. Noskowitz, Phys. Fluids 8, 2337 (1996).

[19] G. Bird, Molecular Gas Dynamics (Oxford University Press, Oxford, 1976); Molecular Gas Dynamics and the Direct Simulation of Gas Flows (Clarendon, Oxford, 1994).

[20] J. J. Brey, M. J. Ruiz-Montero, and D. Cubero, Phys. Rev. E 54, 3664 (1996).

[21] T. P. C. van Noije, R. Brito, and M. H. Ernst (unpublished).

[22] The models in Ref. [8] are formulated for the revised Enskog equation, but the Boltzmann limit follows trivially.

[23] C. Cercignani, Theory and Application of the Boltzmann Equation (Elsevier, New York, 1975); J. Ferziger and H. Karper, Mathematical Theory of Transport Processes in Gases (NorthHolland, Amsterdam, 1972).

[24] I. Goldhirsch and M.-L. Tan, Phys. Fluids 8, 1752 (1996).

[25] R. Zwanzig, J. Chem. Phys. 71, 4416 (1979).

[26] A. Santos, J. J. Brey, and J. W. Dufty, Phys. Rev. Lett. 56, 1571 (1986).

[27] A. Santos and J. J. Brey, Physica A 174, 355 (1991).

[28] D. M. Gass, J. Chem. Phys. 54, 1898 (1971).

[29] S. Chapman and T. G. Cowling, The Mathematical Theory of Non-Uniform Gases (Cambridge University Press, Cambridge, 1970).

[30] J. Gómez Ordóñez, J. J. Brey, and A. Santos, Phys. Rev. A 39, 3038 (1989).

[31] C. S. Campbell, J. Fluid Mech. 203, 449 (1989). 